\title{
Geometrical analysis, for rapid prototyping, of the compressor's helical conic rotor model
}

\author{
Nicuşor Baroiu, Viorel Păunoiu, Virgil Gabriel Teodor ${ }^{*}$, Florin Susac and Nicolae Oancea \\ "Dunarea de Jos" University of Galati, Department of Manufacturing Engineering, Domneasca 111, \\ Galati, Romania
}

\begin{abstract}
The conical rotary compressor represents a solution for gases compression, for relatively small flow, in conditions of a superior energetic efficiency. Although the theoretical solution for the helical conical compressor was elaborated decades ago, a viable technology solution become possible at industrial level only after the development of machine tools with numerically controlled axes. In this paper is proposed a study, analytical and graphical, concerning the geometry of conical rotary compressor's rotors, modelled by rapid prototyping technology. The propose modelling allow to elaborate various constructive forms for conical compressor's rotors.
\end{abstract}

\section{Introduction}

The conical rotary compressor [1] represents a solution for gases compression, in the domain of low flows and high pressures [2]. The constructive solution for such compressor with active elements of the conical helix surfaces has been known for over half a century, but the industrial applications of these constructions become possible, from technological point of view, only after development of machine tools with several numerically controlled axis [1]. The effectiveness for this compressor type was theoretically and experimentally proven, from the energy point of view [2], [3].

An analytical and graphical modelling is proposed in this paper. Also, a 3D model by rapid prototyping for the worms was made.

\section{Analytical form of conical rotary compressor - crossing sections}

For the crossing section of driving rotor of the helical conic compressor is accepted a form composed from an assembly of curves, epicycloids - hypocycloid, see figure 1 . The reference systems are defined: $x y$ is the fixed reference system, with origin onto the rotor's axis, $x_{e} y_{e}$ - auxiliary fixed reference system, joined with the $r_{e}$ roulette axis; $x_{i} y_{i}$ - auxiliary fixed reference system joined with the $r_{i}$ roulette axis; $X_{e} Y_{e}$ - mobile reference system, joined with $r_{e}$ roulette and $X_{i} Y_{i}$ - mobile reference system joined with $r_{i}$ roulette.

${ }^{*}$ Corresponding author: virgil.teodor@ugal.ro 


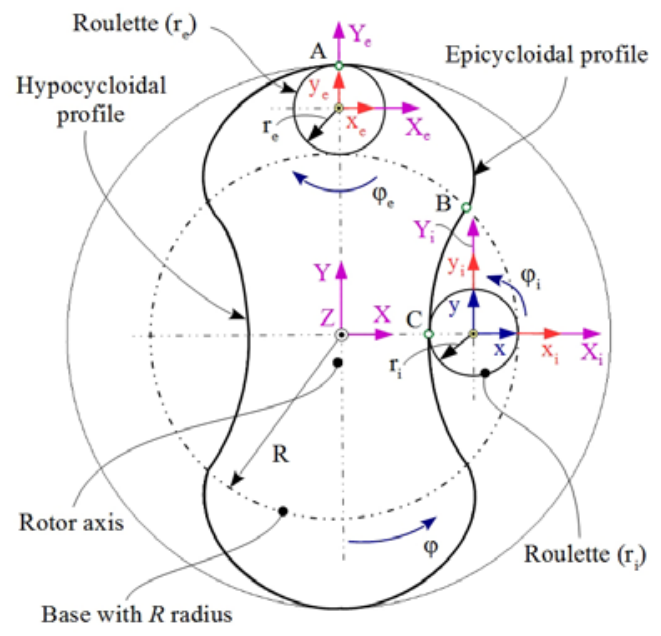

Fig. 1. Crossing profile of helical conic rotor.

The rotor's crossing profile is composed from two cycloid curves, generated by points $A$ and $C$, see figure 1 . The $A$ point has coordinates, in $X_{e} Y_{e}$ Cartesian reference system:

$$
X_{e}=0 ; \quad Y_{e}=r_{e}
$$

And point $C$ has coordinates:

$$
X_{i}=-r_{i} ; \quad Y_{i}=0
$$

The relative motion of the mobiles spaces is given by:

$$
X=\omega_{3}(\varphi)\left[\omega_{3}^{T}\left(-\varphi_{e}\right) \cdot X_{e}+A\right],
$$

With $\left.A=\left(\begin{array}{ll}0 & -\left(R+r_{e}\right.\end{array}\right)\right)^{T}$

After replacements, the equations of the epicycloid in $X Y$ mobile reference system are, for $r_{e}=r_{i}=r$ :

$$
\begin{aligned}
\text { AB } & =r \cdot \sin \left(\varphi+\varphi_{e}\right)-(R+r) \cdot \sin \varphi \\
Y & =r \cdot \cos \left(\varphi+\varphi_{e}\right)-(R+r) \cdot \cos \varphi
\end{aligned}
$$

* From condition $X^{2}+Y^{2}=R^{2},(R-$ basis radius $)$,

The limit for angular parameter results

$$
\varphi_{e}=\operatorname{arcos}\left[\frac{R^{2}-(R+r)^{2}-r^{2}}{2 r \cdot(R+r)}\right] .
$$

** Between $\varphi$ and $\varphi_{e}$ angular parameters exists the relation:

$$
\varphi=(r / R) \cdot \varphi_{e}
$$

Similarly, the $B C$ flank is described. 


\section{Driven worm helical conic flank's equations}

The conic worm assumes a basis with variable radius, $R$, starting from value $R_{0}$ in frontal plane. The results, see also figure 2:

$$
R=R_{0}-2 \cdot p \cdot \varphi_{1} \cdot \operatorname{tg} \alpha
$$

Where $\alpha$ is the cone's generatrix angle; $p$ is helical parameter of conical worm; $\varphi_{1}$ is the twisting angle in helical motion of the conic rotor's crossing profile and $R_{0}$ is basis radius.

We assumes that the roulette radius $r_{e}=r_{i}=r$ is a variable value.

For $r=1 / 4 \cdot R$ results $r=1 / 4 \cdot\left[R_{0}-p \cdot \varphi_{1} \cdot \operatorname{tg} \alpha\right]$, with $R_{0}, \alpha$ and $p$ constructive constants values. In this way, in $X_{l} Y_{l} Z_{l}$ reference system, see figure 2 , the generating motion of helical conic worm is given by:

$$
X_{1}=\omega_{3}^{T}\left(\varphi_{1}\right) \cdot X-p \cdot \varphi_{1} \cdot \vec{k}
$$

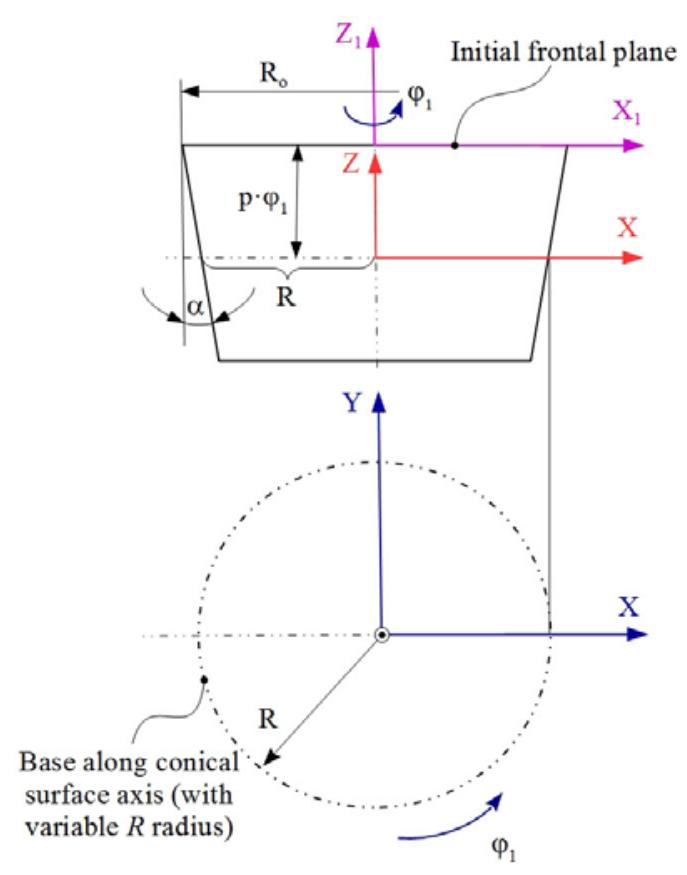

Fig. 2. Conical worm generatrix.

After replacements of cycloid profiles,(4) the $\Sigma_{A B}$ helical conic surface's equations result:

$$
\Sigma_{A B} \mid \begin{aligned}
& X_{1}=\left[r \cdot \sin \left(\varphi+\varphi_{e}\right)-(R+r) \cdot \sin \varphi\right] \cdot \cos \varphi_{1}-\left[r \cdot \cos \left(\varphi+\varphi_{e}\right)-(R+r) \cdot \cos \varphi\right] \cdot \sin \varphi_{1} \\
& Y_{1}=\left[r \cdot \sin \left(\varphi+\varphi_{e}\right)-(R+r) \cdot \sin \varphi\right] \cdot \sin \varphi_{1}+\left[r \cdot \cos \left(\varphi+\varphi_{e}\right)-(R+r) \cdot \cos \varphi\right] \cdot \cos \varphi_{1} \\
& Z_{1}=-p \cdot \varphi_{1}
\end{aligned}
$$

Right hand worm, for conical cycloid flank $A B$. Similarly for the $\Sigma_{B C}$ surface. 


\section{Driven worm flank's equations - crossing sections}

They are defined the reference systems: $X Y Z$ is the mobile reference system, joined with the internal (driving) worm, with origin in $O_{i} ; X_{l} Y_{l} Z_{1}$ - mobile reference system, joined with the external (driven) worm, with origin in $O_{e} ; x_{1} y_{1} z_{1}$ - fixed reference system joined with the external worm axis' base and $x y z$ - fixed reference system, joined with internal worm axis base, see figure 3 . The ratio between $R_{e}$ and $R$ (gearing ratio) is:

$$
i_{12}=\left(R_{e} / R\right)=3 / 2
$$

The angles of rotation motions around the bases axes, $\psi_{1}, \psi_{2}$ :

$$
R_{e} \cdot \psi_{2}=R \cdot \psi_{1} \quad \rightarrow \quad i=\psi_{1} / \psi_{2}=R_{e} / R
$$

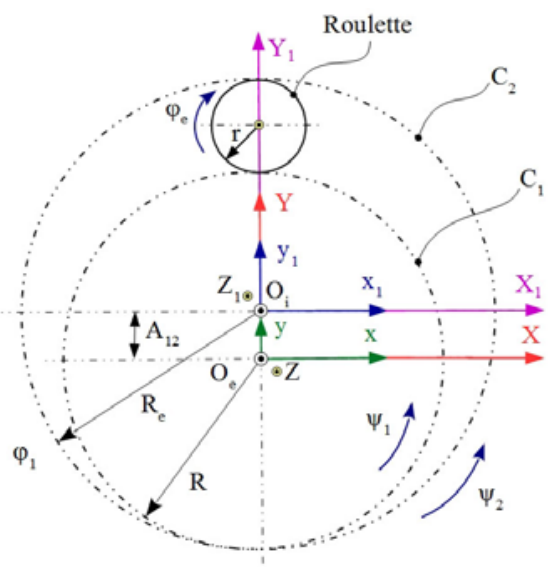

Fig. 3. Rolling centrodes, $C_{I}$ and $C_{2}$ - identically with bases circles with radii $R$ and $R_{e}$ of compressor's rotors, in crossing section.

The relative motion between the two centrodes is defined:

$$
X_{1}=\omega_{3}\left(\psi_{2}\right) \cdot\left[\omega_{3}^{T}\left(\psi_{1}\right) \cdot X+B\right], B=\left(\begin{array}{lll}
0 & -A_{12} & 0
\end{array}\right)^{T} .
$$

If, in (13), the $X$ matrix represents geometric locus of cycloid profiles $A C$, equation (4) , with remark $r_{e}=r$, then transformation (13) represents the cycloid curves' family, in $X_{l} Y_{l} Z_{l}$ reference system. So, from (4) and (13), the surface generated by $A B$ arc results:

$$
\left(\begin{array}{c}
X_{1} \\
Y_{1} \\
Z_{1}
\end{array}\right)=\omega_{3}\left(-\psi_{2}\right) \cdot \omega_{3}^{T}\left(\psi_{1}\right) \cdot\left(\begin{array}{c}
r \cdot \sin \left(\varphi+\varphi_{e}\right)-(R+r) \cdot \sin \varphi \\
r \cdot \cos \left(\varphi+\varphi_{e}\right)-(R+r) \cdot \cos \varphi-A_{12} \\
0
\end{array}\right) \text {, }
$$

In $X_{I} Y_{l} Z_{I}$ reference system joined with compressor's driven worm.

Similarly, for surface generated by $\operatorname{arc} B C$.

Joining these equations with the enwrapping condition specific for one of the complementary methods, for example method of "minimum distance", [4], the profiles in crossing sections of driving worms are determined.

In figure 4, the relative positions between driving and driven worms are presented. 


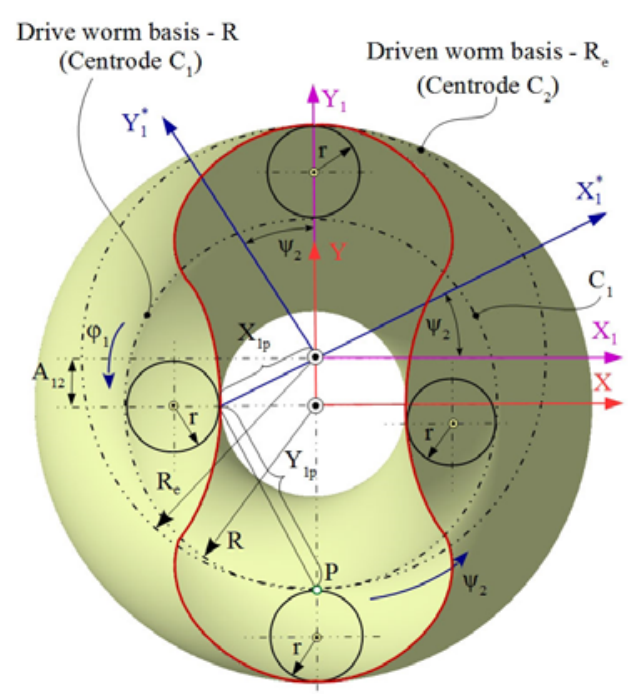

Fig. 4. Rolling centrodes $C_{1}, C_{2}$ - the method of "minimum distance".

In figure 4 , is presented a position (for a value of $\psi_{2}$ angle) of $X_{l} Y_{l} Z_{l}$ reference system, in rolling time. In this position, the coordinates of $P$ gearing pole, in $X_{l} Y_{l} Z_{l}$ reference system, are:

$$
X_{1_{P}}=-R_{e} \cdot \sin \psi_{2} ; Y_{1_{P}}=-R_{e} \cdot \cos \psi_{2} .
$$

In this way, the distance from the current point on the cycloid profile to the gearing pole, expressed in $X_{I} Y_{l} Z_{l}$ reference system is:

$$
d=\sqrt{\left(X_{1}+R_{e} \cdot \cos \psi_{2}\right)^{2}+\left(Y_{1}+R_{e} \cdot \sin \psi_{2}\right)^{2}}
$$

with $X_{1}, Y_{1}$ from (14).The minimum value of this distance is obtained from condition:

$$
d_{\min }=\left(\partial d / \partial \varphi_{1}\right)=0
$$

So, the condition (17) become:

$$
\left(X_{1}+R_{e} \cdot \cos \psi_{2}\right) \cdot X_{\varphi_{1}}+\left(Y_{1}+R_{e} \cdot \sin \psi_{2}\right) \cdot Y_{\varphi_{1}}=0,
$$

Representing the enwrapping condition, specific for the method of "minimum distance", [4].

The (16) and (17) equations assembly, and condition (18) applied for $A B$ and $B C$ profile, see figure 1 , represents the crossing profile of external worm, in $X_{1} Y_{1} Z_{1}$ reference system. Regarding the definition of roulette radius, $r_{e}=r$ and $r_{i}=r$, consecutive sections of driven worm can be determined along the driving worm's axis, see figure 4 .

Also, considering the gearing ratio between the two centrodes with radii $R$ and $R_{i}$,

$$
i_{\psi_{1}, \psi_{2}}=\psi_{2} / \psi_{1}=R / R_{e}
$$




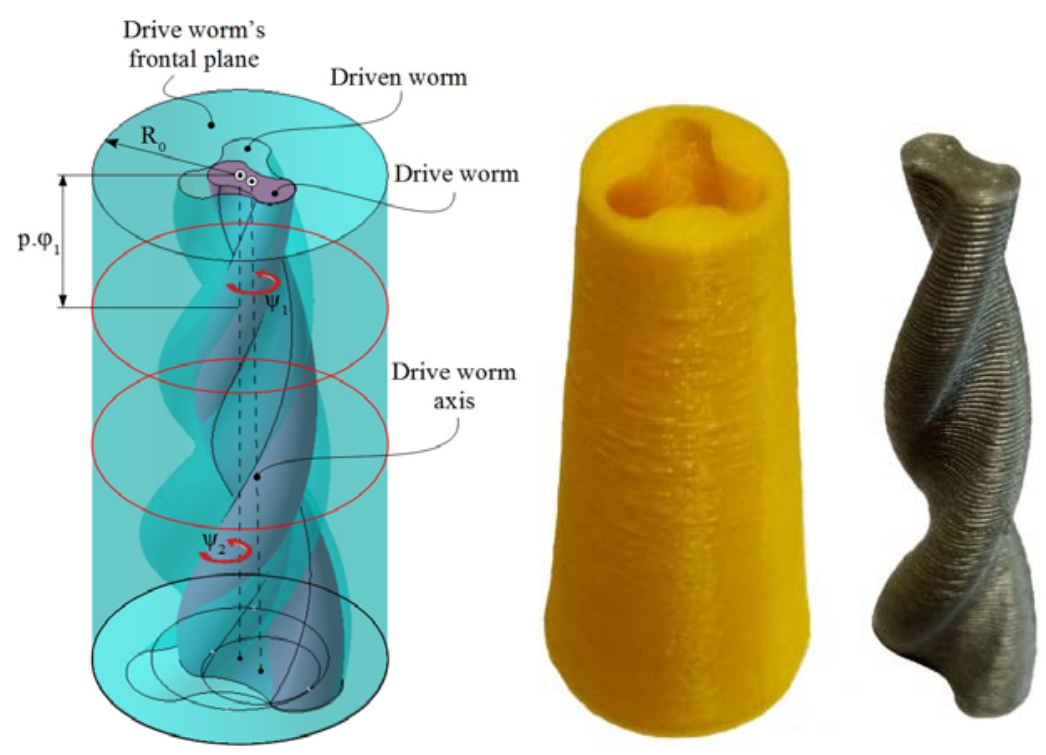

Fig. 5. Positions of three-lobed surface's center against the driving worm's revolving axis; 3D print of the two worms.

\section{Conclusions}

The paper presents the model of a conical cycloid worm determined by analytical geometry. At the same time, based on the complementary theorem of the surface enwrapping, the "minimum distance" method [4], has determined the conjugate worm within the conical helical compressor.

Based on the analytical models of the two worms the solid models of the two worms representing the movable elements of the conical helical compressor were made in CATIA.

These solid models were exported as "stl" file format and were printed on a 3D printer, model Ultimaker3 Extended, using Cura slicing software.

In the future, it is intended to develop an original technology for compressor manufacturing.

\section{References}

1. C. Abagnale, M. Cardone, B. Gargiulo, R. Marialto, Energy Procedia, 101, 710-717 (2016)

2. O. Dmitriev, E. Tabota, EFMC 12, 103-108 (2014)

3. O. Dmitriev, E. Tabota, I. Arbon, IOP Conf. Series: Mat. Science and Eng., 90(2015)

4. N. Oancea, Surfaces generation by winding, (Galati Univ. Press, Galati, 2004) 